\section{Response to Sistermans et al.}

Sistermans et al. are critical of our study that showed that in chorionic villi samples (CVS) from a largely unselected population, only confined placental mosaicism (CPM) involving trisomy 16 was associated with adverse pregnancy outcomes. $^{1,2}$ They assert that our conclusions are not applicable to genome-wide cell-free DNA (gw-cfDNA) screening even though both testing methodologies are based on the cytotrophoblast lineage and identify rare autosomal trisomies (RATs). There are several misrepresentations in Sistermans and colleagues' critique of our study.

They incorrectly state that $72 \%(319 / 443)$ of our CPM involving RATs were lost to follow-up. Over the 18-year study period, the laboratory served numerous centers and, for practical reasons, only 154 RAT cases from 7 representative centers were included. The lost to follow-up rate was 51/154 (33\%). Unavailable follow-up was due to document disposal or patient transfer, which should not lead to bias. The lost to follow-up rate in controls, 289/757 (38\%), was not statistically different from that in CPM cases $(p>0.05)$.

We concluded that an association of RATs with fetal growth restriction (FGR) was spurious. This conclusion was not because the prenatal growth records were incomplete, or because some FGR cases had birthweight percentile $>10$ th. It was because the clinical criteria used by the various participating centers were heterogeneous and they changed over the study period. Moreover, the more clinically relevant and related outcome, birthweight adjusted for gestational age at term, showed no association both as a quantitative and categorical binary variable. We also excluded FGR from the neonatal intensive care unit (NICU) admissions analysis to exclude those cases with medical induced prematurity arising from the prenatal growth concern.

Sistermans et al. ${ }^{1}$ point out that gw-cfDNA and CVS differ because CPMII cases are included the CVS data. We agree this does constitute a difference. However, we observed no associations for pregnancy complications after excluding CPMII. We also acknowledge that the CVS cases in our study excluded cases where amniocentesis confirmed the presence of a RAT but gw-cfDNA analysis would include such cases. Only $3 \%$ of RATs in cytotrophoblasts are confirmed at amniocentesis and not all of these cases will be associated with an abnormal fetus. ${ }^{3}$ Therefore, allowing for this small number of True fetal mosaicism (TFM) cases that would be detected by gw-cfDNA would not materially alter our conclusions regarding the overall utility of the testing in low-risk populations. Our study was not powered to detect weak associations between CPM and adverse outcomes.
To further justify clinical application of gw-cfDNA, Sistermans et al. assert that the level of mosaicism can be assessed through cfDNA measurement and high levels are more likely to have pregnancy complications. Although mosaicism can be detected through gw-cfDNA analysis, ${ }^{4}$ we are unaware of peer reviewed studies showing that the level of mosaicism can be accurately determined. Moreover, the association between level of mosaicism with pregnancy complications has not been established for any of the chromosomes when identified through gw-cfDNA. For any particular trisomy, the observed levels of cfDNA mosaicism in the population are likely to be a continuum with no specific cut-off value defining the group of cases meriting further testing and monitoring. For any individual pregnancy, there is expected to be clinical uncertainty due to possible differences in the proportion of abnormal cells in mesenchyme, trophoblasts, and fetal organs, regardless of the mosaicism level in the cfDNA. ${ }^{5}$ Trisomies of meiotic origin are more likely to involve a high proportion of abnormal cells compared with those of mitotic origin. High levels of trisomy and nonmosaic trisomy are likely to be associated with spontaneous miscarriage but prenatal screening for this purpose is not recommended. ${ }^{6}$

As Sistermans et al. ${ }^{1}$ note, gw-cfDNA can potentially identify large segmental imbalances but these are rare in lowrisk livebirth populations. ${ }^{7,8}$ Even for large imbalances, there can be false positives (for example, due to maternal constitutional or somatic chromosome abnormality) and interpretative complexities for some imbalances. Many segmental imbalances in fetuses will be associated with ultrasound abnormality and for these pregnancies appropriate management includes offering invasive testing with chromosome microarray and possible reflex exome sequencing. Noninvasive prenatal screening (NIPS) is not recommended because it would only delay the definitive genetic diagnosis. Sistermans et al. ${ }^{1}$ claim a high positive predictive value (PPV) for the detection of large imbalances in high-risk populations but they do not provide a PPV for the more clinically relevant group of pregnancies with normal ultrasound findings. Neither do they explain how the benefit of detecting these rare additional imbalances can be realized without also encountering the much higher number of cases with uncertainties associated with a RAT-positive result.

Some, but not all, studies have reported higher levels of adverse outcomes when RATs are identified through gwcfDNA compared with identification through CVS. ${ }^{3}$ These studies also imply that the test would be an inefficient approach to the identification of adverse outcomes and pregnancy complications in a general referral population. Observational studies on selected populations that have heterogeneous referral reasons, differences in the gestational age at testing, follow-up clinical management based on 
currently perceived opinions of the significance, and variable or substantially incomplete follow-up are biased and cannot be expected to provide an unambiguous indication of the clinical significance of this heterogeneous set of chromosome abnormalities. To properly demonstrate the value of gwcfDNA, a randomized clinical trial is needed and clear evidence of improved pregnancy outcomes must be demonstrated. Evidence for acceptability of the protocol by a high proportion of participating women is also essential.

In the Netherlands, women have been enrolled in a study (TRIDENT-2) to evaluate the significance of RATs. Women have been informed through a patient information brochure that they could elect to receive additional information beyond screening for trisomies 21,18 , and 13 and that the clinical significance is severe or serious. ${ }^{9}$ There was no mention that the follow-up testing may be inconclusive, or of the possibility of detecting maternal malignancy, miscarriage, a vanishing twin, or that the finding can be entirely inconsequential.

We believe that the current push to include screening for RATs raises serious ethical and legal challenges because the clinical significance is not sufficiently defined, and women may not be receiving this message prior to their acceptance of gw-cfDNA screening.

\section{DISCLOSURE}

P.B. is a consultant and holds stock options in Natera, Inc. F.R.G. is a full-time employee of TOMA Laboratory, Impact Lab Group without ownership shares, and is an expert panel member for Roche and consultant for Menarini Biomarkers. J.F. declares no conflicts of interest.

Publisher's note Springer Nature remains neutral with regard to jurisdictional claims in published maps and institutional affiliations.
Peter Benn, DSc, PhD ${ }^{1}$, Francesca Romana Grati, $M S c, P h D \mathbb{D}^{2}$ and Jose Ferreira, $\mathrm{MD}, \mathrm{PhD}^{3,4}$

${ }^{1}$ Department of Genetics and Genome Sciences, University of Connecticut Health Center, Farmington, CT, USA; ${ }^{2}$ Unit of Research and Development, Cytogenetics and Medical Genetics TOMA, Advanced Biomedical Assays, Impact Lab Group, Busto Arsizio, Varese, Italy; ${ }^{3}$ Faculty of medicine, Eduardo Mondlane University, Maputo, Mozambique; ${ }^{4}$ Department of Obstetrics and Gynecology, Maputo Central Hospital, Maputo, Mozambique; 5 Genomed S.A., Warsaw, Poland. Correspondence: Francesca Romana Grati (fgrati@tomalab.com)

\section{REFERENCES}

1. Sistermans EA, Van Opstal D, Bekker MN, Pertile MD. The clinical benefit of genome-wide cfDNA testing cannot be extrapolated from CVS data. Genet Med. (in press).

2. Grati FR, Ferreira J, Benn P, et al. Outcomes in pregnancies with a confined placental mosaicism and implications for prenatal screening using cell-free DNA. Genet Med. 2019 Aug 8; https://doi.org/10.1038/ s41436-019-0630-y. [Epub ahead of print].

3. Benn P, Malvestiti F, Grimi B, et al. Rare autosomal trisomies: comparison of detection through cell-free DNA analysis and direct chromosome preparation of chorionic villus samples. Ultrasound Obstet Gynecol. 2019;54:458-467.

4. Brison N, Neofytou M, Dehaspe L, et al. Predicting fetoplacental chromosomal mosaicism during non-invasive prenatal testing. Prenat Diagn. 2018;38:258-266.

5. Taylor TH, Gitlin SA, Patrick JL, et al. The origin, mechanisms, incidence and clinical consequences of chromosomal mosaicism in humans. Hum Reprod Update. 2014;20:571-581.

6. Gregg AR, Skotko BG, Benkendorf JL, et al. Noninvasive prenatal screening for fetal aneuploidy, 2016 update: a position statement of the American College of Medical Genetics and Genomics. Genet Med. 2016;18:1056-1065.

7. Benn P. Expanding non-invasive prenatal testing beyond chromosomes 21, 18, 13, X and Y. Clin Genet. 2016;90:477-485.

8. Benn P, Grati FR. Genome-wide non-invasive prenatal screening for all cytogenetically visible imbalances. Ultrasound Obstet Gynecol. 2018;51:429-433.

9. Information about the prenatal screening for Down syndrome, Edwards' syndrome and Patau's syndrome. Prenatal screening May 2019. https:// www.rivm.nl/documenten/folder-informatie-over-screening-op-downedwards-en-patausyndroom-engels-english. Last accessed 27th October 2019.

Advance online publication 4 November 2019. doi:10.1038/s41436-019-0687-7 